\title{
Syndrome of transtentorial herniation: is vertical displacement necessary?
}

\author{
Allan H Ropper
}

\begin{abstract}
MRI from a comatose patient with a massive acute subdural haematoma showed most of the features of transtentorial herniation described in the classic pathology literature. In addition to encroachment on the perimesencephalic cisterns, infarction in the anterior and posterior cerebral artery territories, ischaemic change in the lower diencephalon, and ventricular enlargement were visualised. Despite the clinical syndrome and these secondary changes due to compression, there was only approximately $2 \mathrm{~mm}$ of downward displacement of the upper brainstem compared with 13 mm horizontal displacement. Although tissue shifts adjacent to the tentorial aperture cause brainstem and vascular compression, these changes may occur with minimal downward herniation.
\end{abstract}

$(\mathcal{F}$ Neurol Neurosurg Psychiatry 1993;56:932-935)

In the presence of a large intracranial mass the true relationships of structures near the tentorial notch may not be appreciated as well by postmortem examination as they are by radiological analysis during life. The CT and MRI features of herniation from a unilateral mass have been reported, ${ }^{1-4}$ and several MRI scan measurements have been proposed to detect downward movement of the upper brainstem, ${ }^{5-7}$ but rarely with clinical correlation. This paper demonstrates most of the known primary and secondary radiological features of transtentorial herniation by MRI scans from a comatose patient with a large acute subdural haematoma. The relative vertical and horizontal displacements of the upper brainstem were measured in an advanced stage of herniation to determine the relative contributions of each.

St Elizabeth's

Hospital, Boston, MA,

USA

A H Ropper

Correspondence to:

Dr Ropper, St Elizabeth's

Hospital, 736 Cambridge St

Boston, MA 02135, USA.

Received 17 August 1992

and in revised form

25 January 1993

Accepted 29 January 1993 motionless and the left side had extensor posturing. There were bilateral Babinski's signs.

\section{Case report}

As 86 year old woman fell at home and was found unresponsive. On admission she was comatose, the left pupil was $6 \mathrm{~cm}$, and right pupil $4 \mathrm{~mm}$ in diameter, both round and
She had no oculocephalic eye movements. A CT scan showed a large left sided subdural haematoma with $13 \mathrm{~mm}$ of horizontal shift of the pineal calcification from the midline, and the perimesencephalic cisterns were obliterated. The MRI scan described below was performed 22 hours later, with the same signs present. She died four days after admission. Necropsy was not obtained.

\section{MRI findings}

Axial, coronal, and sagittal T-1 weighted images were obtained. Axial scans just below the plane of the tentorium (fig 1) showed convex distortion and extreme displacement of the upper brainstem away from the subdural clot, and medial temporal tissue in the perimesencephalic cistern on the side of the mass. The upper pons showed less displacement but the trigeminal nerve on the side of the mass was stretched (fig 2). Coronal images showed the distortion of the entire

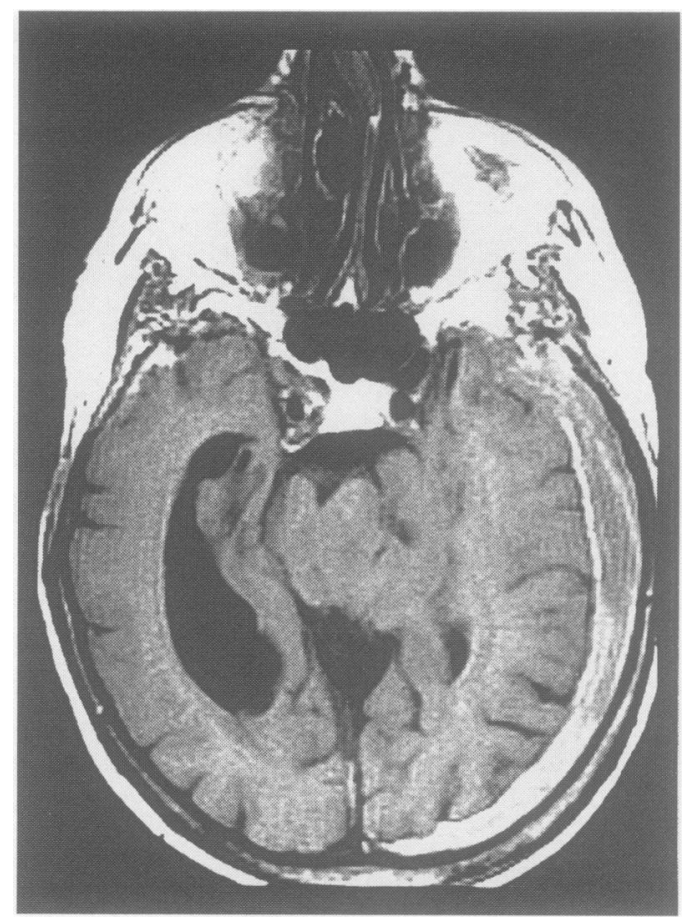

Figure 1 Axial MRI, $5 \mathrm{~mm}$ thick, $T-1$ weighted, TR 720/TE 15, showing the left uncus and parahippocampal gyrus within the perimesencephalic cistern. There is upper brainstem displacement and distortion, and enlargement of the contralateral ventricle. 

the level of the upper pons showing displacement of the mass, stretching of the trigeminal nerve on the side of the clot, and slackening contralaterally. The sixth nerves could not be visualised.

Figure 3 Coronal T-1 weighted images showing the convex displacement away from the clot and medial temporal lobe herniation.
Figure 2 Axial MRI at the brainstem away from
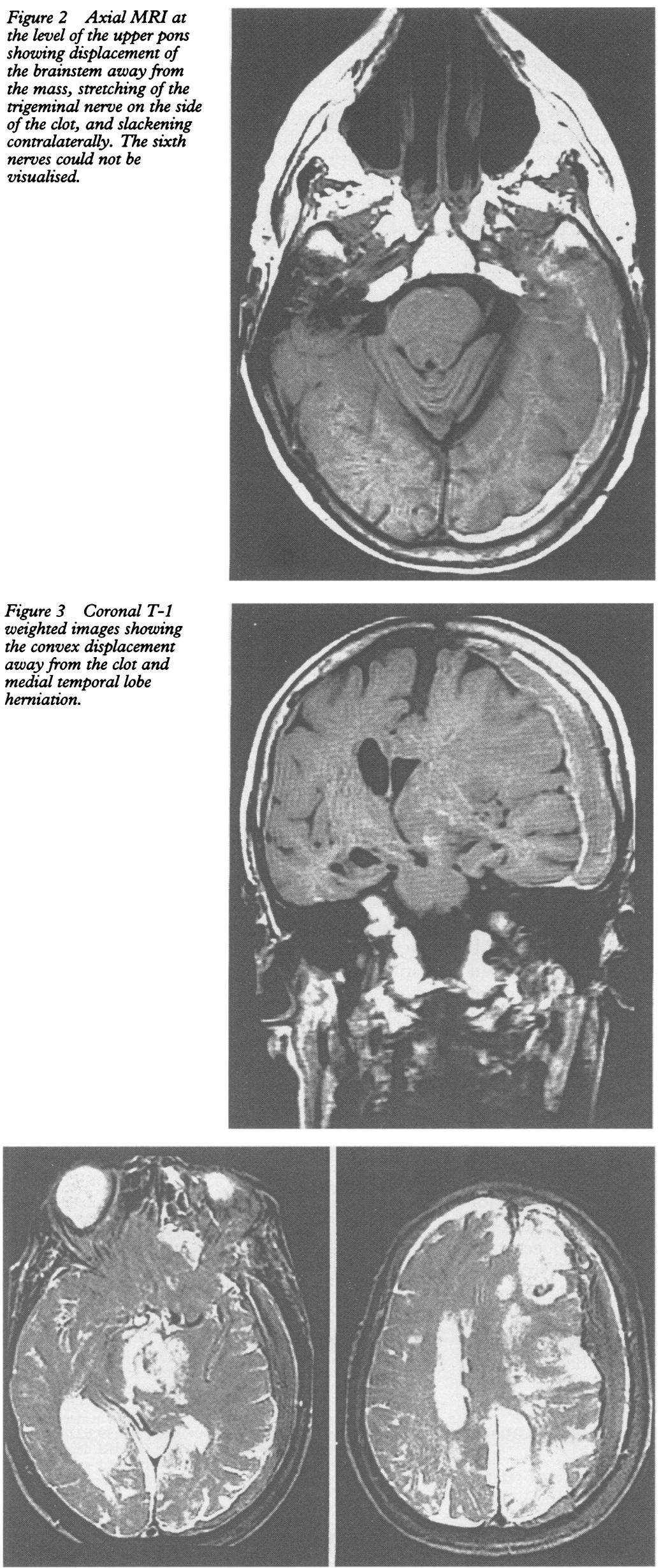

Figure 4 Axial T-2 weighted images, TR 3000/TE 80 demonstrating infarctions in the upper brainstem and in the regions of the anterior and posterior cerebral arteries on the side of the mass and a small area of medial occipital infarction opposite the mass. central brain region with temporal lobe encroachment on the cerebral peduncle (fig $3)$. Left cingulate gyrus infarction was also demonstrated. Coronal scans showed the deep tissue shifts best, including the encroachment of the left temporal lobe on the adjacent perimesencephalic cistern, but there was a large degree of displacement of the midbrain-diencephalic region above this level, leaving unclear the role of the herniated tissue in causing brainstem displacement. The occipital ventricular horn contralateral to the clot was enlarged.

T-2 weighted images showed infarction of the lower diencephalon and upper midbrain, and infarctions in the territories of the posterior cerebral artery on the side of the clot, of the calcarine branch opposite the clot, and of the callosomarginal branch of the anterior cerebral artery (fig 4).

\section{Horizontal and vertical measurements}

Measurements were made on printed MRI as previously described using electronic calipers, ${ }^{6}$ and confirmed by direct computer measurements on the MRI screen. There was less than $10 \%$ difference in measurements made by the two techniques.

Sagittal images and vertical displacement: On a mid-sagittal T-1 weighted MRI, the pontmesencephalic junction (PMJ) was $5 \mathrm{~mm}$ above Twining's line in true perpendicular distance (fig 5), therefore representing approximately $2 \mathrm{~mm}$ downward movement compared with normal subjects from Feldman's series ${ }^{5}$ and our own normal subjects. The ratio of the vertical perpendicular distance between Twining's line and PMJ to the length of Twining's line, a measurement utilised by Feldman et al, ${ }^{5}$ was $0 \cdot 042$, midway between the normal subjects and patients with masses and higher than their awake

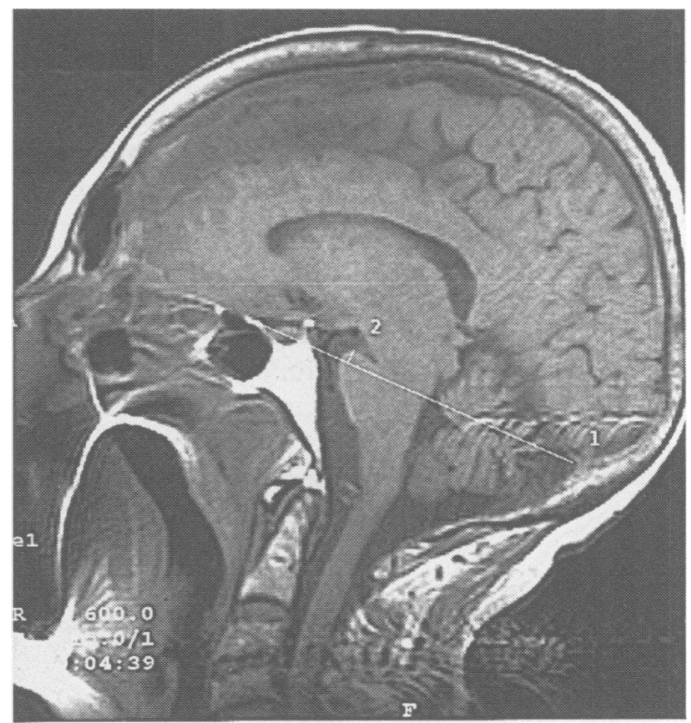

Figure 5 Sagittal T-1 weighted image with (1)

Twining's line and (2) the perpendicular distance to the pontomesencephalic junction of $5 \mathrm{~mm}$ indicated $(2 \mathrm{~mm}$ below its normal position). The iter of the aqueduct cannot be clearly seen because of displacement out of the plane of the image, but it is situated less than $5 \mathrm{~mm}$ below the incisural line used by Reich, et al . 
patients with masses. This ratio again represents a true dimension of approximately 1-2 $\mathrm{mm}$ downward displacement of the PMJ (length of Twining's line was $10 \mathrm{~cm}$ ). An improved reference measurement for the displacement of the posterior midbrain, introduced by Reich et al, ${ }^{7}$ uses the displacement of the iter (upper opening of the aqueduct) from its normal position on a line approximating the plane of the incisura. This could not be measured with precision because the aqueduct was compressed and the iter was displaced horizontally out of the mid-sagittal plane (as it is in most patients with masses). An approximate measurement for this vertical displacement was less than $5 \mathrm{~mm}$ downward.

Coronal images and horizontal displacement: On coronal images (fig 3) there was $13 \mathrm{~mm}$ horizontal displacement of the lowest point of the third ventricle from the midline true dimension (the same as pineal displacement on (T), and $37.8 \mathrm{~mm}$ vertical distance from the pontomesencephalic junction (PMJ) to the cerebral midline point, representing $1 \cdot 2$ $\mathrm{mm}$ depression of the PMJ below its normal position compared with controls in a previous study. ${ }^{6}$

The three sagittal measurements and one coronal measurement of vertical displacement therefore converged to estimate approximately $2 \mathrm{~mm}$ downward displacement of the upper brainstem structure compared to 13 $\mathrm{mm}$ lateral displacement of structures in that region. The technically less precise measurement between the iter and the incisural line suggested, at most, $5 \mathrm{~mm}$ downward displacement of the posterior brainstem.

\section{Discussion}

MRI can now be performed on acutely ill patients and may give more insight than necropsy studies into the pathoanatomy of herniation. Almost all of the features of transtentorial herniation due to a large unilateral mass that are known from pathology studies could be appreciated in the MRI scans in this case. Accompanying these changes was, however, extreme horizontal but little axial downward displacement of the upper brainstem near the plane of the tentorial incisura. Infarctions in both the posterior cerebral artery territories, ${ }^{8-14}$ and collosomarginal branch of the anterior cerebral artery $^{111516}$ territory were detected with T-2 weighted images. Extreme signal changes occurred in the compressed diencephalicmesencephalic junction, ${ }^{17}$ including the radiological features of the Kernohan's notch phenomenon. ${ }^{18}$ These changes in the upper brainstem presumably reflected damage in the reticular activating system that caused coma.

In analysing the regional anatomy of structures near the tentorium, the advanced stage of tissue displacement and preceding 22 hours of coma must be considered. Transtentorial herniation of the medial temproal lobe had occurred by the time of the MRI scan but it is difficult to know if this degree of tissue distortion was necessary for coma and pupillary changes. There was a large uncal herniation on the side of the mass and the ipsilateral pupil was $6 \mathrm{~mm}$ in diameter, the opposite, $4 \mathrm{~mm}$, but without uncal herniation on that side. These scans do not settle the precise relationship of herniation to upper brainstem compression. The parahippocampal gyrus may not be the proximate cause of compression and displacement of the upper brainstem; rather it may move into the perimesencephalic cistern passively as the entire hemisphere is displaced horizontally above. ${ }^{619}$ Although there were points of contiguity between the cerebral peduncle and the displaced medial temporal lobe, the incisural cistern could still be visualised on coronal scans, suggesting that the horizontal brainstem displacement resulted from tissue shifts well above the tenorial plane, and enlargement of the cistern may have been a passive phenomenon.

There was concordance between horizontal shift of the pineal on CT scan and of the lower third ventricular shift on MRI scan. Midline structures such as the pontomesencephalic junction and aqueduct are moved out of the mid-sagittal plane by large hemispheral masses, making most measurements in these patients only approximations. The newer reference line introduced by Reich et $a l$ is an improvement from Twining's line but compression and displacement of the aqueduct also limits its use when there is horizontal displacement. These inaccuracies are compounded by the optical problems in calibrating measurements of this small size. As discussed previously, ${ }^{6}$ it is difficult to compare the physiological effects on upper brainstem neurons of small downward displacement compared with larger horizontal displacements. Nevertheless, even in the advanced stage of medial temporal lobe herniation in our patient with coma, pupillary changes, and bilateral compression of major vessels at the tentorial aperture, there was little downward "herniation" of the upper brainstem, contrasted with substantial horizontal displacement.

1 Osborn AG. Diagnosis of transtentorial herniation by cranial computed tomography. Radiology 1977;123:93-6.

2 Stovring J. Descending tentorial herniation: Findings on computed tomography. Neuroradiology 1977;14:101-5.

3 Nguyen JP, Djindjian M, Brugieres P, Badiane S, Melon E, Poirer J. Correlations anatomo-scanographiques dans les engagements cerebreaux transtentoriels. $f$ dans les engagements cerebrear

4 Hahn FJ. Signs of central descending transtentorial herniation. AFNR 1985;6:844-5.

5 Feldman E, Gandy SE, Becker R, Zimmerman R, Thaler HT, Posner JB, Plum F. MRI demonstrates descending transtentorial herniation. Neurology 1988;38:697-701.

6 Ropper AH. A preliminary MRI study of the geometry of brain displacement and level of consciousness with acute intracranial masses. Neurology 1989;39:622-7.

7 Reich JB, Sierra J, Deck MDF, Plum F. MRI description and clinical correlation of dynamic upward and downward transtentorial herniation. Neurology 1991;41 (suppl):390-1.

8 Sato M, Tanaka S, Kohama A, Fujii C. Occipital lobe infarction caused by tentorial herniation. Neurosurgery 1986;18:300-5.

9 Marinkovic SV, Milisavljevic MM, Lolic-Dragnic V, Kovacevic MS. Distribution of the occipital branches of the posterior artery. Correlation with occipital lobe the posterior artery. Correlation

10 Blinkov SM, Gabibov GA, Tanyashin SV. Variations in 
location of the arteries coursing between the brainstem and the free edge of the tentorium. $f$ Neurosurgery 1992;76:973-8.

11 Lindenberg $R$. Compression of brain arteries as pathogenetic factor for tissue necroses and their areas of predilection. F Neuropathology Exp Neurology 1985;14: 223-30.

12 Evans JP, Scheinker IM. Histologic studies of the brain following head trauma. III. Post traumatic infarction of cerebral arteries, with consideration of the associated clinical picture. Arch Neurology Psychiatry 1943;50: 258-78.

13 Moore MT, Stern K. Vascular lesions in the brain-stem and occipital lobe occurring in association with brain and occipital lobe occurring

14 Sunderaland $S$. The tentorial notch and complications produced by herniations of the brain through that aper- ture. Brit $\mathcal{Y}$ Surgery 1958;45:422-38.

15 Rothfus WE, Goldberg AL, Tabas JH, Deeb ZL. Callosomarginal infarction secondary to transfalcial herniation. AFNR 1987;8:1073-6.

16 Sohn D, Levine S. Frontal lobe infarctions caused by brain herniation. Compression of the anterior cerebral artery branches. Arch Pathology Lab Medicine 1967;84: 509-12.

17 Niikawa S, Uno T, Ohkuma A, Hara A, Nokura H, Yamada $\mathrm{H}$. Occlusion of a perforating artery indicating descending tentorial herniation after head injury, supplying deep cerebral structure-report of 4 cases and their CT evaluation. No To Shinkei 1988;40:1151-6.

18 Cohen AR, Wilson J. Magnetic resonance imaging of Kernohan's notch. Neurosurgery 1990;27:205-7.

19 Fisher CM. Acute brain herniation: a revised concept. Seminars in Neurology 1984;4:417-21. 